\title{
Effect of Type of Metals on Site Selective Dehydrogenation of Stearic Acid
}

\author{
Chayakorn KARASUTA $^{1 *}$, Supakorn SIRIMITTAGOON ${ }^{2}$, Ratchanon CHANTANUSON ${ }^{2}$ and Varong PAVARAJARN ${ }^{1}$ \\ ${ }^{1}$ Department of Chemical Engineering, Faculty of Engineering, Chulalongkorn University, 254 Phayathai Rd, Wang Mai, Pathum \\ Wan District, Bangkok 10330, Thailand \\ ${ }^{2}$ Green Technology Research Co., Ltd. 16 ${ }^{\text {th }}$ Floor, AIA Capital Center Building 89 Ratchadaphisek Rd., Dindaeng, Bangkok 10400 , \\ Thailand
}

\begin{abstract}
Short-chain fatty acids (SCFAs) have been used as raw materials in wide range of chemical and medical applications. One technique to produce SCFAs is oxidative cleavage of long-chain fatty acids (LCFAs). However, unless the LCFAs are unsaturated, the yield of SCFAs is often very low because the carboxylic group of the fatty acid is more active than other part of the molecule. This work explores the idea of introducing a double bond into saturated LCFA, i.e., stearic acid, via selective dehydrogenation using commercial heterogeneous catalysts. However, cracking of the LCFA is also catalysed. Different type of metals was therefore investigated to study the effect of metals on the cracking and dehydrogenation. The experiments were conducted in an autoclave reactor under inert atmosphere. The temperature was in the range of $250-350^{\circ} \mathrm{C}$. The products were analysed by gas chromatography equipped with mass spectroscopy (GC/MS). The results reveal that the introduction of double bond in the aliphatic chain of the stearic acid is possible although the yields of the unsaturated LCFAs are low. Effects of various parameters, such as temperature, pressure, and reaction time, were also investigated and reported.
\end{abstract}

\section{Introduction}

Nowadays, demands of fatty acids (FAs) have been increased in many industries, especially for SCFAs. SCFAs are not abundantly available in nature. Although SCFAs can be obtained from hydrolysis of both plant and animal fats, the yield if naturally small. Many processes have been developed to synthesize SCFAs, i.e., from petroleum or from palm oil, but the processes are usually complicate and require high production cost. Moreover, most process could not control the chain length of FAs as needed, and the main products are deoxygenated product instead.

In general, FAs contain a carboxylic functional group, which is relatively active compared to aliphatic tail. Thus, most of the reactions done on FAs takes place on this functional group instead of on the aliphatic tail as expected. Nevertheless, a recent study demonstrated that an oxidative cleavage can lead to specific cracking at the location of double bond on the long aliphatic chain of FAs, leading to FAs with shorter hydrocarbon chain (Enferadi et al., 2015). Thereby, specific SCFAs could be obtained from saturated fatty acids (SFAs), For saturated FAs, however, they should be dehydrogenated to introduce a double bond into their aliphatic chain first. Nevertheless, previous studies have stated that dehydrogenation of aliphatic tail requires multistep sequences to navigate the reactivity of the carboxylic functionality (Reich et al.,
1973; Trost et al., 1976). By using an allyl-palladium homogeneous catalyst, a site-selective dehydrogenation is possible, but it is successful only for introducing a double bond at $\alpha$ and $\beta$ location (Zhao et al., 2017; Szewczyk et al., 2018).

For heterogeneous catalyst, Snare et al. revealed that an aliphatic tail dehydrogenation could be done like a homogeneous catalyst by using bifunction catalysts (Snåre et al., 2006; Berenblyum et al., 2012). These catalysts are comprised of dehydrogenation component, often a noble metal, and acid site on a support (Weitkamp, 2012). These findings are also in line with the study of Wang et al. that the main function of noble metals is to dehydrogenate substrate molecules (Simakova et al., 2009; Na et al., 2010). Although a noble metal could perform dehydrogenation, it still depends on structure and functional group within a compound to be dehydrogenated. That is, the key step might be an adsorption step on the surface of the catalyst (Simonetti et al., 2012; Simonetti et al., 2013). This work investigate possibility of using noble metals in site-selective dehydrogenation of saturated LCFA, i.e., stearic acid, on alumina support.

\footnotetext{
Corresponding author: chayakorn.kar@gmail.com
} 


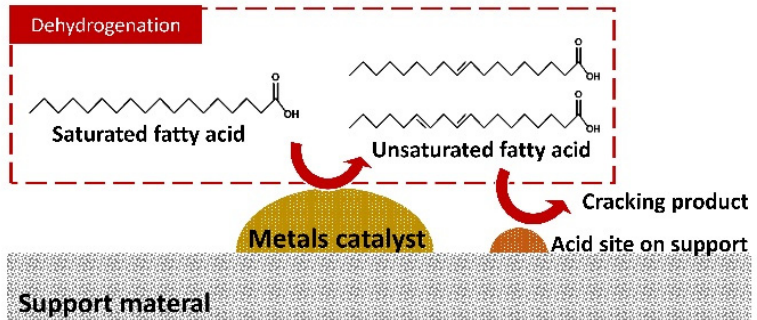

Figure 1. Schematic diagram for interaction between a substrate and metal catalyst.

\section{Experimental}

\subsection{Materials}

Stearic acid $\left(\mathrm{C}_{18} \mathrm{H}_{36} \mathrm{O}_{2}, 98 \%\right.$, synthesis grade, PanReac Applichem), sodium hydroxide $(\mathrm{NaOH}$, Analytic reagent, Ajax Finechem Pty LTd), methanol $\left(\mathrm{CH}_{3} \mathrm{OH}, \mathrm{AR}\right.$ grade, QRec), methyl stearate $\left(\mathrm{C}_{19} \mathrm{H}_{38} \mathrm{O}_{2}, 10 \mathrm{mg} / \mathrm{ml}\right.$ in hexane, AccuStandard), methyl oleate $\left(\mathrm{C}_{19} \mathrm{H}_{36} \mathrm{O}_{2}, 1000 \mu \mathrm{g} / \mathrm{ml}\right.$ in hexane, AccuStandard), standard $\mathrm{C}_{8}-\mathrm{C}_{20}$ alkane $(10 \mathrm{mg} / \mathrm{L}$ in hexane, Sigma-Aldrich), hydrochloric acid ( $\mathrm{HCl}, 1.0 \mathrm{~N}$, $\mathrm{ACl}$ labscan) and sodium sulphate anhydrous (Analytic reagent grade, Fisher chemical) were used as received.

\subsection{Preparation of the catalyst}

The $\mathrm{Pt} / \mathrm{Al}_{2} \mathrm{O}_{3}$ catalyst was purchased from Sigma-Aldrich, while; the commercial $\mathrm{Pd} / \mathrm{Al}_{2} \mathrm{O}_{3}$ and $\mathrm{Ni} / \mathrm{Al}_{2} \mathrm{O}_{3}$ with the same loading was obtained from Liaoing Haitai Sci-Tech Development Co., Ltd. To reduce the catalysts, the catalysts were dried at $120^{\circ} \mathrm{C}$ overnight in an oven. Then, they were reduced under a mixture of hydrogen and nitrogen.

\subsection{Characterization of the catalyst}

The specific surface area (BET) and pore volume of the noble metals on alumina support were determined using the nitrogen adsorption isotherms in volumetric adsorption systems (Micromeritics 3 flex surface characterization analyzer).

\subsection{Catalytic dehydrogenation of stearic acid}

Stearic acid and reduced catalyst were added into a stainless autoclave reactor equipped with a controller. Then, it was purged and pressurized by nitrogen gas. The reactor was then heated. Since, this work was a new idea to introduce of double bond into aliphatic tails, as the beginning, it was focus on only the effect of temperature (range of 250 to $350^{\circ} \mathrm{C}$ ) and types of metals (Pt, Ni and $\mathrm{Pd}$ on supported alumina) on site-selective dehydrogenation of stearic acid, while; the other parameters, such as reaction time and pressure, was fixed
(5 hrs of operation and 30 bar). The sample was periodically collected through a needle valve. Moreover, the gas from the reactor was also collected into a gas bag for GC-FID analysis.

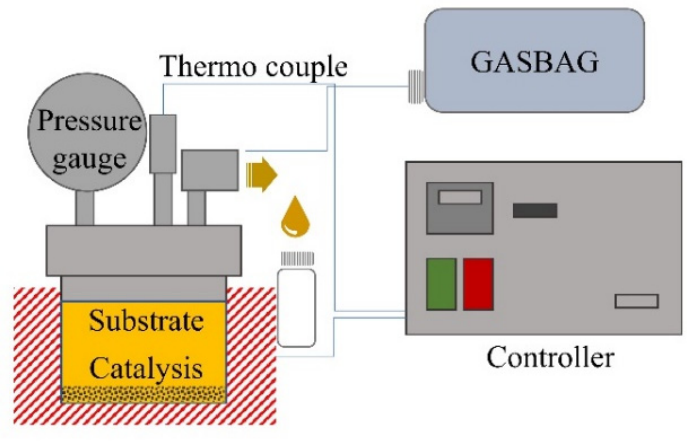

Figure 2. Reactor with controller.

\subsection{GC-MS analysis of the products}

The products were also analysed by GC-MS (Shimadzu QP2020) with HP-INNOWAX column $(30 \mathrm{~m} \times 0.25 \mathrm{~mm}$ $\mathrm{x} 0.25 \mu \mathrm{m})$. However, they were esterified prior to the injection via a procedure proposed by Ichihara and Fukubayashi (2010). The heating rate was set at $3^{\circ} \mathrm{C} / \mathrm{min}$, starting from $50^{\circ} \mathrm{C}$ to $260^{\circ} \mathrm{C}$ and held for $5 \mathrm{~min}$. The injector split ratio was set at 10:1. The mass spectrometer detector was operated with the ion source temperature of $260^{\circ} \mathrm{C}$ and interface at $260^{\circ} \mathrm{C}$, with $\mathrm{m} / \mathrm{z}$ in range of 35 400 in scan mode. The product was identified by NIST14 data library with probability equal to or higher than $90 \%$. Standard methyl stearate, methyl oleate, and $\mathrm{C}_{8}-\mathrm{C}_{20}$ alkane were used for quantitative analysis.

Note that, the calculation of total unsaturated fatty acid $\mathrm{C}_{18}$ was based on calibration curve of oleic acid for identification the unsaturated molecule of fatty acid. The definition of the conversion, selectivity and yield are summarized below.

$$
\begin{gathered}
\% \text { Conversion }=\frac{\text { mole of stearic acid comsumed }}{\text { mole of stearic acid before reaction }} \times 100 \\
\% \text { Selectivity }=\frac{\text { mole of total unsaturated fatty acid } C_{18}}{\text { mole of stearic acid consumed }} \times 100 \\
\% \text { Yield }=\frac{\% \text { Conversion } \times \% \text { Selectivity }}{100}
\end{gathered}
$$

\section{Results \#}

\subsection{Characterization of the catalysts}

\subsubsection{Texture properties of catalysts}

The nitrogen adsorption/desorption isotherms of the noble metal/alumina catalysts and alumina support were presented in Figure 3. The hysteresis loop of catalysts exhibits cylinder-shaped pores. The specific surface area, total pore volume and average pore were calculated, and are listed in Table 1. The $\mathrm{Pd} / \mathrm{Al}_{2} \mathrm{O}_{3}$ and $\mathrm{Ni} / \mathrm{Al}_{2} \mathrm{O}_{3}$ commercial catalysts were insignificantly different, in 
textural properties, while a $\mathrm{Pt} / \mathrm{Al}_{2} \mathrm{O}_{3}$ catalyst has significantly lower specific surface area.

Table 1. Texture properties of catalysts.

\begin{tabular}{lccc}
\hline Catalyst & $\begin{array}{c}\text { BET } \\
\text { Surface } \\
\text { area } \\
\left(\mathrm{m}^{2} / \mathrm{g}\right)\end{array}$ & $\begin{array}{c}\text { Pore volume } \\
\left(\mathrm{cm}^{3} / \mathrm{g}\right)\end{array}$ & $\begin{array}{c}\text { Pore size } \\
\text { diameter }(\mathrm{nm})\end{array}$ \\
\hline $\mathrm{Al}_{2} \mathrm{O}_{3}$ & 299.4 & 0.5767 & 7.7 \\
$\mathrm{Pt} / \mathrm{Al}_{2} \mathrm{O}_{3}$ & 96.5 & 0.2207 & 9.2 \\
$\mathrm{Pd} / \mathrm{Al}_{2} \mathrm{O}_{3}$ & 244.15 & 0.5339 & 8.7 \\
$\mathrm{Ni} / \mathrm{Al}_{2} \mathrm{O}_{3}$ & 261.3 & 0.5410 & 8.3 \\
\hline
\end{tabular}

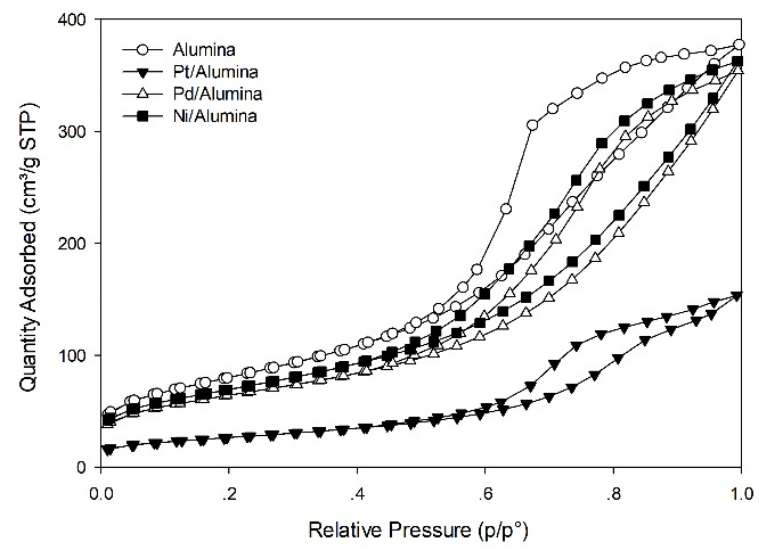

Figure 3. Nitrogen adsorption-desorption \#sotherms for catalyst.

\subsection{Catalytic results}

Dehydrogenation of saturated LCFAs involves hydrogen elimination/dehydrogenation which removes hydrogen from the molecule of FAs. This process could occur at both carboxylic part and aliphatic tail of the FAs. Since the carboxylic functional group is more reactive than the aliphatic tail, the reaction at the aliphatic tail is more difficult. To overcome this restriction, it was hypothesized based on the ability of noble metals heterogeneous catalyst in the dehydrogenation of longchain alkanes (LCAs), which has been proposed in literatures (He et al., 2009; He et al., 2015; He et al., 2016). Platinum, Palladium, and Nickel on Alumina support have been reported to shown catalytic activity toward dehydrogenation of paraffin (Sattler et al., 2014; Zhang et al., 2015).

Note that, Figure 4 to 5 were a relative plot that all of data were a ratio which divided by a numeric constant due to terms and conditions of the collaborated company. The results were found that performances of difference catalysts are significantly different, especially in conversion at high temperature. The conversion is especially low in absence of the catalyst, as shown in Figure 4. The yields of the unsaturated LCAs are shown in Figure 5. It is shown in Figure 6 and 7 that, as the temperature was raised, the contents of both hydrogen and carbon dioxide in the autoclave are increased. Carbon monoxide was increased with the increase in temperature only on Pd-based catalyst and in non-catalytic condition, as shown in Figure 8. Comparing Figure 5 with Figure 6, the increase in hydrogen content in gas phase incorporates the reduction in both selectivity and yield of unsaturated LCFAs due to reversible process on noble metals site. This behaviour was not observed in the reaction without catalyst. Pdbased catalyst yields lower amount of unsaturated LCFAs than Pt-based catalyst. Although the yields are low, Figure 5 still suggests that introduction of a double bond in the aliphatic tail of stearic acid is possible. It should be noted that the position of the introduced double bond varies, as witnessed from various kinds of C18:1 fatty acid detected, as shown in Table 3. However, as the temperature is higher than $300^{\circ} \mathrm{C}$, the dehydrogenation could take place without the catalyst, which is consistent with the report in literature (Bhasin et al., 2001). The yield of unsaturated LCFAs from the catalytic dehydrogenation at high temperature is decreased because the metal may also catalyse the hydrogenation. This is supported by the fact that hydrogen was increased at high temperature. The increase in hydrogen may also cause hydrodeoxygenation. The reaction at high temperature also result in decarboxylated products which can be noticed from an increase in carbon dioxide and $\mathrm{C}_{17}$ alkane formation, as shown in Figure 7. In addition, methane $\left(\mathrm{CH}_{4}\right)$ was detected from both of catalytic and noncatalytic processes, which suggests the presence of carbon monoxide methanation, in particularly at high temperature (Berenblyum et al., 2012). However, this behavior was not occurred on pure-alumina catalyst. It was a good support for this hypothesis that the role of metal act as the dehydrogenated or hydrogenated sites while the supports are not. Thus, the pure-alumina was not detected an unsaturated fatty acid compound from purealumina and also the content of hydrogen in autoclave reactor, as shown in Figure 5 and 6.

At low temperature, the main products are longchain alkane, i.e., $\mathrm{C}_{15}, \mathrm{C}_{16}, \mathrm{C}_{17}$ alkane (see Table 2). The formation of $\mathrm{C}_{17}$ alkane is significant as high temperature. Besides, other products were also found at the temperature higher than $300^{\circ} \mathrm{C}$, i.e., short-chain alkanes and $\mathrm{C}_{18}$ alkane. This might result from the decrease in thermal stability of long-chain hydrocarbon as high temperature (Bhasin et al., 2001; He et al., 2009).

Table 2. Percent selectivity/yield of decarboxylated product C17.

\begin{tabular}{|c|c|c|c|c|c|c|c|c|c|c|}
\hline \multirow{2}{*}{$\begin{array}{l}\text { Temp. } \\
\text { (C) }\end{array}$} & \multicolumn{2}{|c|}{ Blank } & \multicolumn{2}{|c|}{ Alumina } & \multicolumn{2}{|c|}{$\mathrm{Pt}$ /Alumian } & \multicolumn{2}{|c|}{$\mathrm{Pd}$ /Alumina } & \multicolumn{2}{|c|}{$\mathrm{Ni}$ /Alumina } \\
\hline & $\%$ Selec. & $\%$ Yield & $\%$ Selec. & $\%$ Yield & $\%$ Selec. & $\%$ Yield & $\%$ Selec. & \%Yield & $\%$ Selec. & $\%$ Yield \\
\hline 250 & $1.13 \pm 0.06$ & $0.04 \pm 0.00$ & $0.39 \pm 0.02$ & $0.05 \pm 0.02$ & $97.50 \pm 4.87$ & $11.14 \pm 0.56$ & $40.68 \pm 2.03$ & $5.10 \pm 0.26$ & $2.78 \pm 0.14$ & $0.46 \pm 0.02$ \\
\hline 300 & $2.28 \pm 0.11$ & $0.16 \pm 0.01$ & $0.30 \pm 0.02$ & $0.05 \pm 0.02$ & $75.03 \pm 3.75$ & $10.21 \pm 0.51$ & $24.63 \pm 1.23$ & $6.49 \pm 0.32$ & $5.10 \pm 0.26$ & $1.07 \pm 0.05$ \\
\hline 330 & $30.17 \pm 1.51$ & $1.81 \pm 0.09$ & $0.82 \pm 0.04$ & $0.17 \pm 0.09$ & $60.80 \pm 3.04$ & $17.29 \pm 0.86$ & $65.61 \pm 3.28$ & $22.50 \pm 1.13$ & $5.46 \pm 0.27$ & $0.93 \pm 0.05$ \\
\hline 350 & $25.46 \pm 1.27$ & $4.57 \pm 0.23$ & $2.41 \pm 0.12$ & $0.59 \pm 0.03$ & $48.05 \pm 2.40$ & $22.22 \pm 1.11$ & $48.22 \pm 2.41$ & $27.09 \pm 1.35$ & $16.57 \pm 0.83$ & $4.17 \pm 0.21$ \\
\hline
\end{tabular}




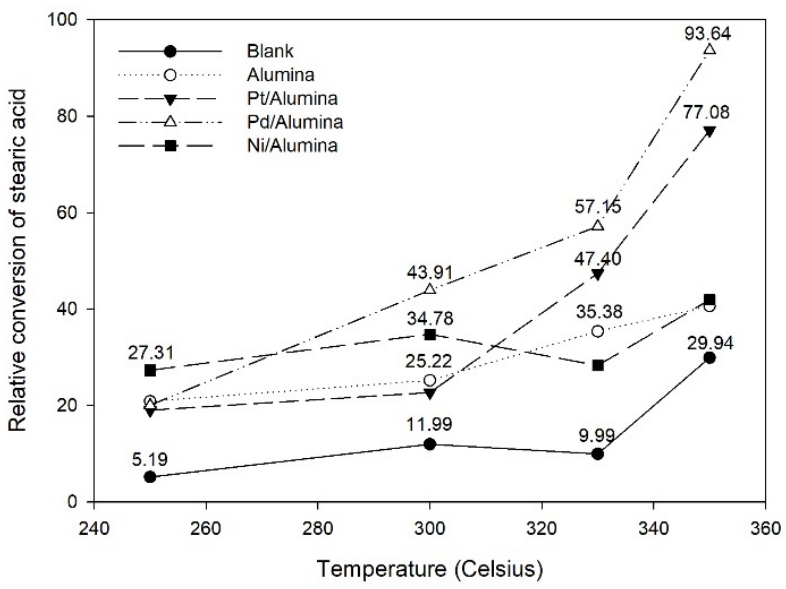

Figure 4. Effect of temperature and catalysts on conversion.

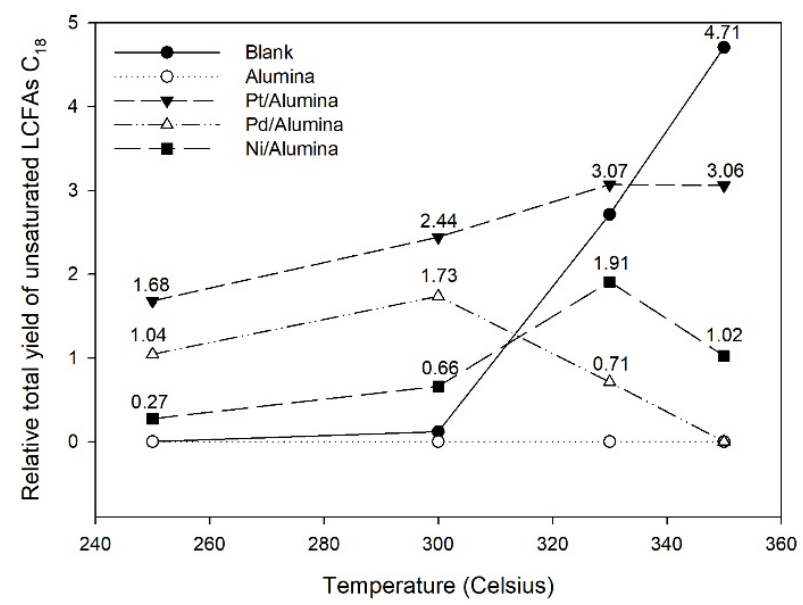

Figure 5. Effect of temperature and catalysts on total unsaturated LCFAs yield.

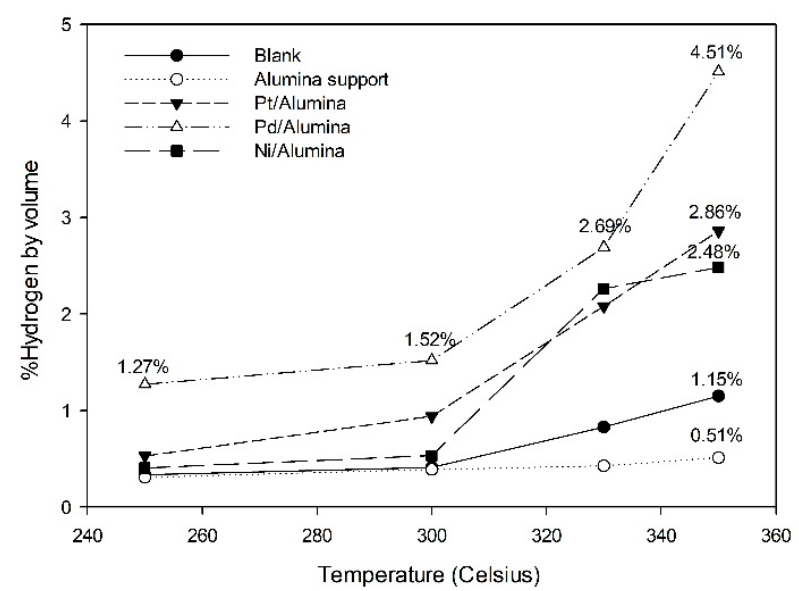

Figure 6. Percent of hydrogen occurred from the reaction.

Although both dehydrogenation/hydrogenation and defunctionalisation could be catalysed by a bifunctional catalyst, decarbonylation are mainly occurred in this case, as evidenced from the trend of $\mathrm{C}_{17}$ selectivity as shown in Table 2. On the other hand, alumina employed as the support has acidic sites that could accelerate a lot of unexpected reactions from unsaturated molecule, e.g., skeletal isomerization, cracking, oligomerization and polymerization (Bhasin et al., 2001; He et al., 2009; Lai,
He et al., 2014; Sattler et al., 2014). In addition, these hypotheses were also in line with $\mathrm{Na}$ et al. ( $\mathrm{Na}$ et al., 2010). It has been reported that decanoic acid mainly occurs from cracking of oleic acid when a catalyst with high acidity was employed. Since the unsaturated LCFAs are reactants for many subsequent reactions, the yield of the unsaturated LCFAs is typically low. The tentative pathway of reactions is shown in Figure 9. Other kinds of compounds such as alcohol, aldehyde, ketone, and alkene were also detected as products in this work.

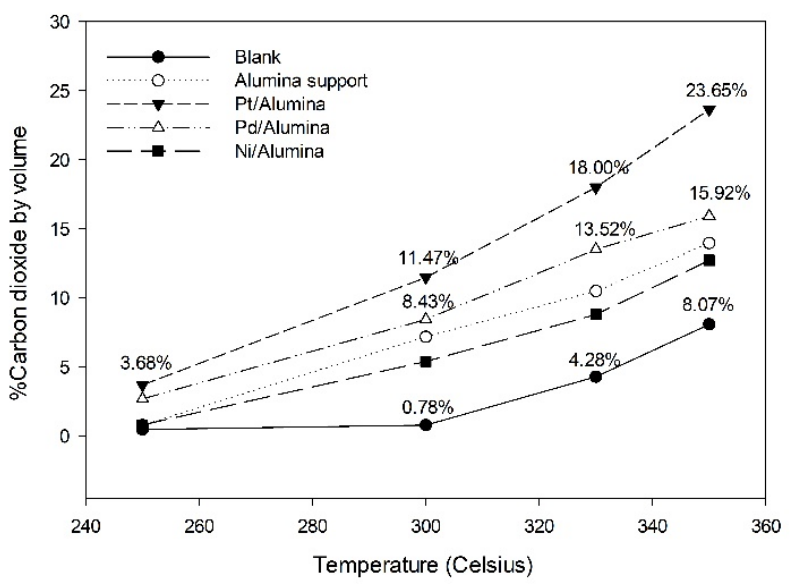

Figure 7. Percent of carbon dioxide occurred from reaction.

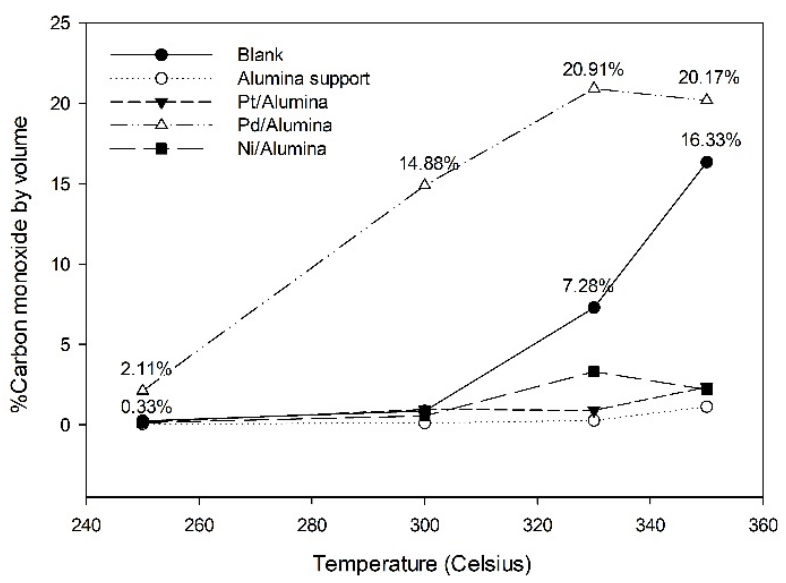

Figure 8. Percent of carbon monoxide occurred from reaction.

Table 3. An unsaturated compound founded in product.

\begin{tabular}{lc}
\hline \multicolumn{1}{c}{ IUPAC name } & Lipid numbers \\
\hline 4-Octadecenoic acid & $\mathrm{C} 18: 1$ \\
6-Octadecenoic acid $(\mathrm{Z}, \mathrm{E})^{*}$ & $\mathrm{C} 18: 1$ \\
8-Octadecenoic acid & $\mathrm{C} 18: 1$ \\
9-Octadecenoic acid $(\mathrm{Z}, \mathrm{E})^{*}$ & $\mathrm{C} 18: 1$ \\
11-Octadecenoic acid & $\mathrm{C} 18: 1$ \\
10-Octadecenoic acid & $\mathrm{C} 18: 1$ \\
13-Octadecenoic acid (Z, E)* & $\mathrm{C} 18: 1$ \\
14-Octadecenoic acid & $\mathrm{C} 18: 1$ \\
15-Octadecenoic acid & $\mathrm{C} 18: 1$ \\
16-Octadecenoic acid & $\mathrm{C} 18: 1$ \\
9, 12-Octadecanotic acid $(\mathrm{Z}, \mathrm{Z})$ & $\mathrm{C} 18: 2$ \\
\hline
\end{tabular}

*(Z,E): cis- and tran- fatty acid which can detect 


\section{Conclusions}

This study explores the new idea of improving FAs, especially the synthesis of SCFAs form saturated LCFAs. Unless the LCFAs are unsaturated, the SCFAs is often difficult to obtain. The results revealed that noble metals on alumina support have potential to introduce double bond in the aliphatic tail of the stearic acid, but the yields of the total unsaturated LCFAs are low. This might be result from acidity of support which causes cracking of the unsaturated molecule. Type of noble metal and range of temperature also affect the distribution of the products. Hence, the support of catalysts should be modified in order to suppress the effect of supported acidity, while it should retain the interaction with the metal without effecting the aliphatic tail interaction.

As for the furthermore studies, it should to study the effect of reaction time and pressure on site-selective dehydrogenation.

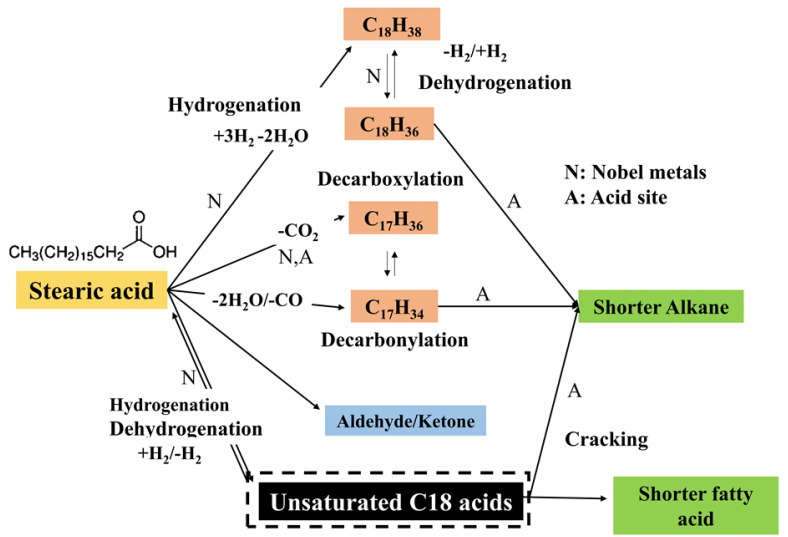

Figure 9 A tentative reaction pathway of stearic acid over Noble metals on alumina support.

\section{References}

Berenblyum, A. S., T. A. Podoplelova, E. A. Katsman, R. S. Shamsiev, and V. Ya. Danyushevsky; "Kinetics and Mechanism of the Deoxygenation of Stearic acid in the Presence of Palladium Catalysts on Alumina," ICCOS, 53(5), 595-609 (2012)

Bhasin, M. M., J. H. McCain, B. V. Vora, T. Imai, and P. R. Pujadó; "Dehydrogenation and Oxydehydrogenation of Paraffins to Olefins," Appl Catal., A., 221(1), 397-419 (2001)

Enferadi Kerenkan, A., F. Béland, and T.-O Do; "Catalytic Oxidative Cleavage of Unsaturated Fatty Acids and Their Derivatives into Valuable Products for Industrial Applications," Catal. Sci. Technol., 6, 971-987 (2015)

He, S., J. Li, B. Wang, X. Dai, C. Sun, Z. Bai and K. Seshan; "Metals Modification of the Paraffin Dehydrogenation Catalyst Regenerated by Coke Combustion," Appl Catal., A., 513, 82-88 (2016)
He, S., C. Sun, Z. Bai, X. Dai, and B. Wang; "Dehydrogenation of Long Chain Paraffins Over Supported Pt-Sn-K/ $/ \mathrm{Al}_{2} \mathrm{O}_{3}$ Catalysts: A Study of the Alumina Support Effect," Appl Catal., A., 356(1), 88-98 (2009)

He, S., B. Wang, X. Dai, C. Sun, Z. Bai, X. Wang, and Q. Guo; "Industrial Development of Long Chain Paraffin (n$\mathrm{C}_{10}-\mathrm{C}_{13}$ ) Dehydrogenation Catalysts and the Deactivation Characterization," Chem. Eng. J., 275, 298-304 (2015)

Lai, Y., S. He, X. Li, C. Sun and K. Seshan; "Dehydrogenation of $n$-Dodecane over $\mathrm{PtSn} / \mathrm{MgAlO}$ Catalysts: Investigating the Catalyst Performance while Monitoring the Products," Appl Catal., A., 469, 74-80 (2014)

Na, J.-G., B. E. Yi, J. N. Kim, K. B. Yi, S.-Y. Park, J.-H. Park, and C. H. Ko; "Hydrocarbon Production from Decarboxylation of Fatty Acid without Hydrogen," Catal. Today, 156(1), 44-48 (2010)

Reich, H. J., I. L. Reich, and J. M. Renga; "Organoselenium Chemistry Alpha-Phenylseleno Carbonyl Compounds as Precursors for Alpha, Beta.Unsaturated Ketones and Esters," J. Am. Che,. Soc., 95(17), 5813-5815 (1973)

Sattler, J. J. H. B., J. Ruiz-Martinez, E. Santillan-Jimenez, and B. M. Weckhuysen; "Catalytic Dehydrogenation of Light Alkanes on Metals and Metal Oxides," Chem. Rev., 114(20), 10613-10653 (2014)

Simakova, I., O. Simakova, P. Mäki-Arvela, A. Simakov, M. Estrada, and D. Y. Murzin; "Deoxygenation of Palmitic and Stearic Acid over Supported Pd Catalysts: Effect of Metal Dispersion," Appl Catal., A., 355(1), 100-108 (2009)

Simonetti, S., S. Ulacco, G. Brizuela, and A. Juan; "Computational Study of Cis-Oleic Acid Adsorption on Ni(1 1 1) Surface," Appl. Surf. Sci., 258(15), 5903-5908 (2012)

Simonetti, S., M. Martirena, S. Ulacco, and G. Brizuela; "Platinum-Nickel Catalyst: The Effect of Promoters in Cis-Oleic Acid Adsorption," Appl. Surf. Sci., 264, 305311 (2013)

Snåre, M., I. Kubičková, P. Mäki-Arvela, K. Eränen, and D. Y. Murzin; "Heterogeneous Catalytic Deoxygenation of Stearic Acid for Production of Biodiesel," Ind. Eng. Chem. Res., 45(16), 5708-5715 (2006)

Szewczyk, S. M., Y. Zhao, H. A. Sakai, P. Dube, and T. R. Newhouse; “ $\alpha, \beta$-Dehydrogenation of Esters with Free $\mathrm{OH}$ and $\mathrm{NH}$ Functionalities via Allyl-Palladium Catalysis," Tetrahedron, 74(26), 3293-3300 (2018)

Trost, B. M., T. N. Salzmann, and K. Hiroi; "New Synthetic Reactions Sulfenylations and 
Dehydrosulfenylations of Esters and Ketones," $\mathrm{J}$. Am. Chem. Soc., 98(16), 4887-902 (1976)

Weitkamp, J; "Catalytic Hydrocracking-Mechanisms and Versatility of the Process," ChemCatChem, 4(3), 292 $-306(2012)$

Zhang, Y., W. Yao, H. Fang, A. Hu and Z. Huang; "Catalytic Alkane Dehydrogenations," Sci. Bull., 60(15), 1316-1331 (2015)

Zhao, Y., Y. Chen, and T. R. Newhouse; "AllylPalladium-Catalyzed $\alpha, \beta$-Dehydrogenation of Carboxylic Acids via Enediolates," Angew. Chem., 56(42), 1312213125 (2017) 